\section{Cultural and Ethical Challenges in Providing Palliative Care for Cancer Patients at the End-of-Life}

\author{
Karima Elshamy, DNSc ${ }^{*}$ \\ Department of Medical Surgical Nursing, Faculty of Nursing, Mansoura University, Mansoura, \\ Dakahlia Governorate 35516, Egypt
}

\section{ABSTRACT}

End-of-life (EOL) care presents many challenges both for clinicians, as well as for patients and their families. Moreover, the care of the dying patient must be considered within the context of the psychological, physical, and social experiences of a person's life. Foremost among those who require EOL care are cancer patients at the EOL. Unfortunately, clinicians who are responsible for the treatment of patients at the EOL commonly lack adequate training to help guide EOL decisions and to deliver bad news to patients and families. They must also face their own discomfort with discussions about death and deal with poor compensation for the time spent discussing EOL care with patients and families. Given the unique process of each person's death, strategies are often inadequate to guide patients, their families, and the clinicians who care for them through this complex and emotionally challenging process. It would stand the clinician in good stead to be aware of these challenges and have an approach towards dealing with such problems. In addition, organizations have a responsibility to ensure that systems are in place to minimize its occurrence and ensure that staff are supported through the process of resolving issues and conflicts that may arise.

KEY WORDS: Culture; Ethical; Challenges; Palliative care; Cancer patients; End-of-life (EOL).

\section{INTRODUCTION}

Cultures vary across countries in terms of economic status, education and resources, as well as by traditional and family values, and religious or spiritual aspects pertaining to illness and health. Cultural beliefs and values shape our approach to death and dying, and need to be acknowledged and included in the delivery of palliative care services, it also provides us with a framework for understanding death, and give meaning to our experiences of suffering and loss. Cultural background has a significant role in coping with the difficult situations at the endof-life (EOL), where health professionals, specially nursing staff and social workers, may be very helpful. Cultural beliefs impact on many aspects of EOL care, including the disclosure of information, decision-making, the use of life-prolonging treatments, and the experience of grief and bereavement. Our traditional practices around death are often linked to our beliefs about the meaning of death and what lies beyond, and have both emotional and spiritual significance. ${ }^{1,2}$ Furthermore, some cultural aspects of death and terminal patients' care should be considered while providing palliative care for those patients in our country. We should pay attention and focus on cultural and social aspects because the whole family is usually involved in a patient's problems as family ties are very strong among people. Understanding medical, ethical, legal and economical challenges which may be experienced by physicians and other cancer care providers in our healthcare system in the special sociocultural context will help in providing an appropriate care for cancer patients at EOL.

Cancer is an illness that affects large numbers of people and their families in Egypt; and the burden of this life-threatening condition have caused many challenging problems for 
the patients, their families, and the public health system in the country, it is a major cause of mortality and morbidity worldwide. In fact, cancer, being a life-threatening disease, makes the inevitable need for the implementation of an effective EOL care strategy in healthcare services more than ever. ${ }^{3}$ Physicians should consider the patients' best interests; and the treatment they choose should be beneficial to the patients and should not do any harm. Moreover, the treatment should be selected on the basis of the patients and their families' views on EOL care and financial costs of terminal care. ${ }^{4}$ Oncologists can fairly decide on EOL provided that they know patients' and their families' values and preferences, ${ }^{5}$ and can communicate with them honestly. ${ }^{6}$ Many physicians avoid informing patients of their survival chance, because they believe that discussing this issue may diminish patient's hope. ${ }^{7}$ In addition, healthcare costs are high in many healthcare units and financial discussions about the cost of treatments and care of end stage cancer patients can be of paramount importance. ${ }^{8}$ Consequently, considering differences of healthcare services in various cultural backgrounds and social structures, the professionals in service providing face with numerous ethical problems for decision-making. ${ }^{9,10}$

EOL care is challenging and can be influenced by cultural and religious values. The specific practices at the last moments of life differ among the religious sectors. It is also important to note that individuals within the same community may have different levels of observance for religious or cultural practices. Consequently, healthcare professionals must be prepared for a diversity of attitudes, values, and practices of dying and bereaved persons. Religions consider death to be a transition from one form of being to another: 'death is a natural end for humans.' Life on earth for a Christian, Muslim, and Jew is an examination and an opportunity to be prepared for life after death, where one's deeds on earth will be judged and accordingly people will be sent to either Heaven or Hell. To optimize our care, we must ensure that social and cultural aspects of life and death are identified, embraced and understood by health professionals so that the needs of patients and their families are met as they approach death. Planning and preparation will ensure that practitioners understand different cultural perceptions of dying and death and respect patients' belief systems and cultural norms. ${ }^{11,12}$

\section{Egypt' Health Profile (Who Statistical Profile)}

World Health Organization - Non-communicable diseases (NCD) Country Profiles, 2014 (Table 1), (Figures 1 and 2).

\begin{tabular}{|c|c|}
\hline Total population (2015) & $91,508,000$ \\
\hline Gross national income per capita (PPP international \$, 2013) & 10 \\
\hline Life expectancy at birth m/f (years, 2015) & $69 / 73$ \\
\hline Probability of dying under five (per 1000 live, 0 ) & Not available \\
\hline Probability of dying between 15 and 60 years $\mathrm{m} / \mathrm{f}$ (per 1000 population, 2015) & $196 / 119$ \\
\hline Total expenditure on health per capita (Intl \$, 2014) & 594 \\
\hline Total expenditure on health as \% of GDP (2014) & 5.6 \\
\hline
\end{tabular}

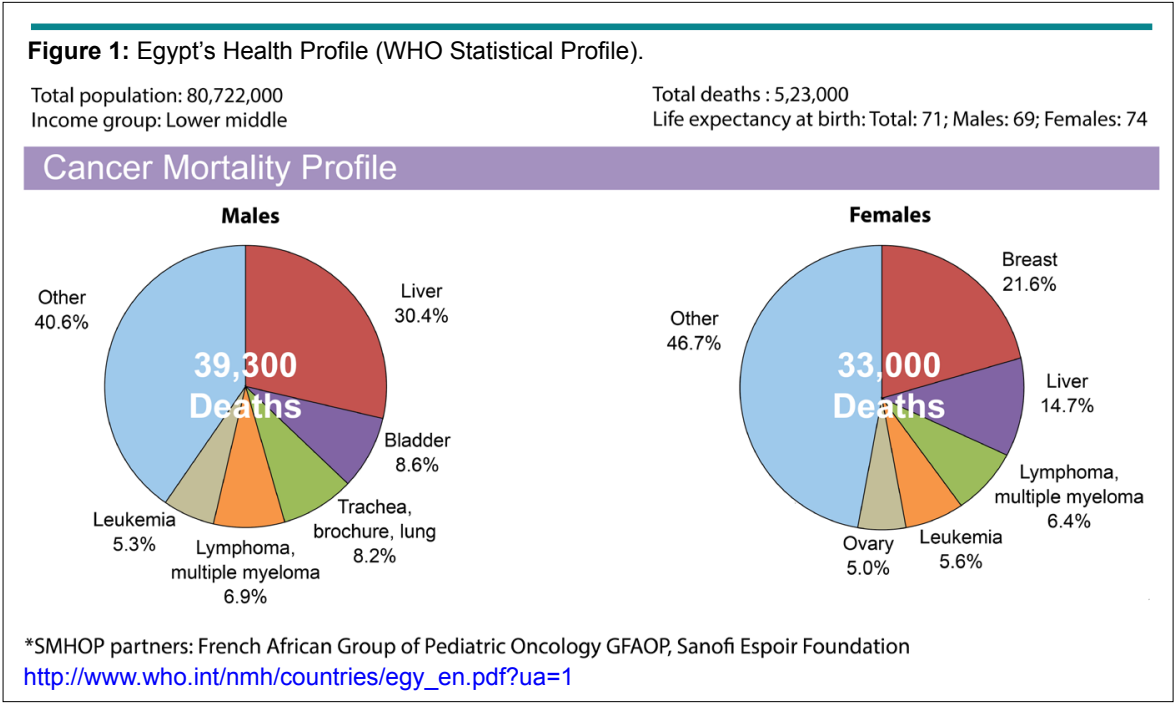




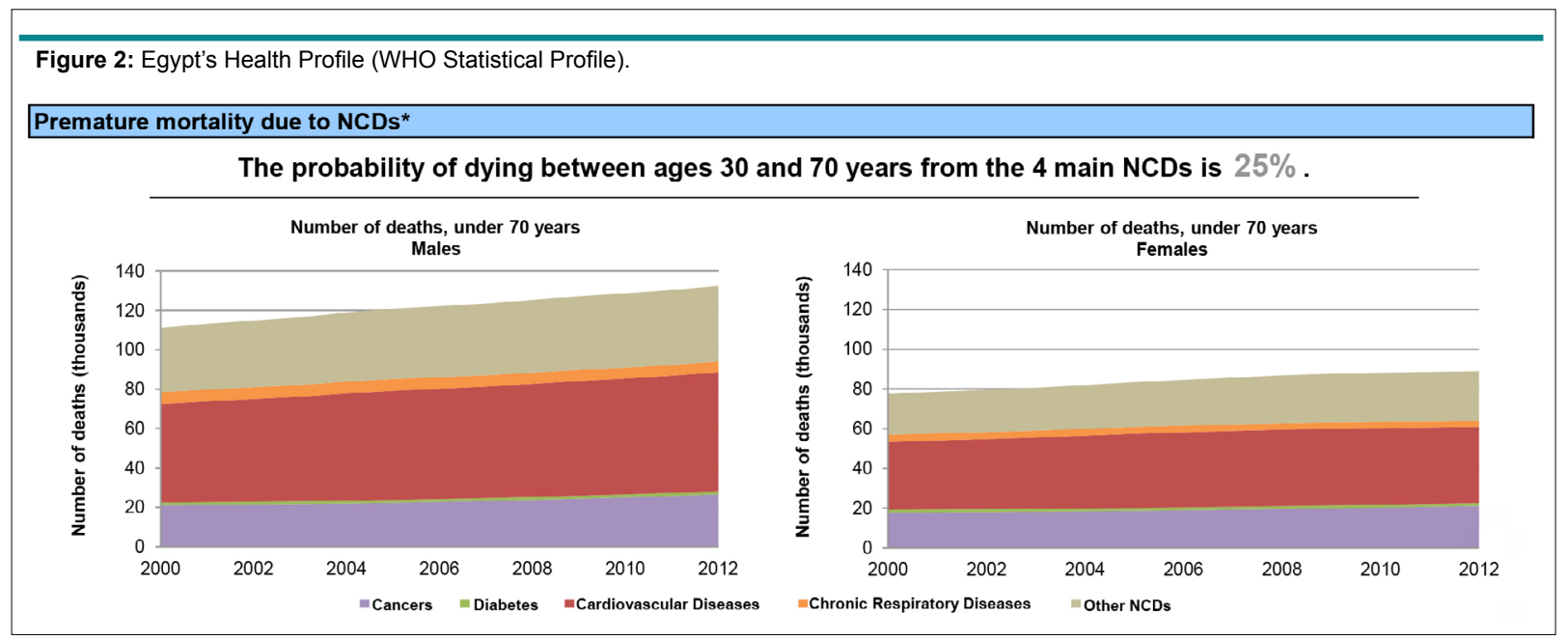

\section{An Overview of EOL Care}

\section{Common Approaches to EOL Care}

Hospice care: Dying patients may choose hospice care. A holistic and philosophical approach to EOL care, hospice brings doctors, nurses, social workers and other professionals together as a care team. The hospice team's goal is to make the patient as comfortable as possible during his or her final days. Hospice emphasizes pain control, symptom management, natural death, and quality of life (QOL) to comfort the patient's physical body. ${ }^{13}$ The hospice team cares for the dying patient wherever that patient is: at home, in a nursing home, in a hospital, or in a separate hospice facility. In addition to medical care, the hospice team may provide emotional and spiritual support, social services, nutrition counseling, and grief counseling for both the patient and loved ones.

Palliative care: Palliative care works to achieve one of the primary goals of healthcare relief of symptoms. Palliative care is an option for patients who are seriously or terminally ill. It focuses on achieving the best possible QOL for a patient by emphasizing on over all comprehensive care for all of patient's needs: pain and symptom management, spiritual, social, psychological, and emotional well-being. ${ }^{14}$

Palliative care is similar to that of hospice care. One striking similarity between hospice and palliative care is the use of an interdisciplinary team of professionals including doctors, nurses, social workers, psychologists, chaplains, and others to provide comprehensive care.

Traditional American medical practice focuses primarily on curing illnesses and healing injuries. Symptom relief is often a secondary focus. ${ }^{15}$ Palliative care supporters believe that failing to address the suffering of a patient with a terminal illness violates two of the main ethical principles behind healthcare. ${ }^{16}$

1) Providing help or benefit to a patient (beneficence) - Failing to relieve pain and other symptoms does not help the dying patient.

2) Not harming a patient (non-maleficence) - Failing to relieve pain and other symptoms can actually harm a patient and the patient's loved ones. For dying patients, palliative treatment provides relief of suffering from pain and other symptoms.

\section{Most Common Symptoms in Dying Patients}

Dying patients frequently experience significant suffering in form of difficulty in breathing, pain, and depression, as:

Difficulty in breathing: Nearly $75 \%$ of people who are imminently dying, experience dyspnea, or "air hunger.". ${ }^{17}$

Pain: Research finds that the number of seriously ill patients who experience substantial pain ranges from $36 \%$ to $75 \%{ }^{18}$

Depression: In a representative study, about 1 in 4 patients admitted to a palliative care unit, mention depression as a significant symptom. ${ }^{19}$

\section{Common Elements of a Good Death}

- Adequate pain and symptom management.

- Avoiding prolonged dying process.

- Clear communication about decisions with patients, family and physician.

- Adequate preparation for death of the patient, for both patient and family.

- Feeling a sense of control.

- Finding a spiritual or emotional sense of completion.

- Affirming the patient as a unique and worthy person.

- Strengthening relationships with loved ones.

- Not being alone. 


\section{Challenges due to Considerations of Patients' Illness and their Families involved with EOL Care}

Cultural background is one of the most important issues in providing cancer care. Social and cultural issues affect the patients' care very much, and they are very important. Based upon our culture, many families do not allow their patients to be aware of the nature and severity of their disease. Therefore, the patient has no active role in decision making. Cancer patients towards their EOL know from the physicians that the disease is not curable, and they are not treated for overcoming the disease to increase their survival, but they are only treated to improve their QOL, and thus the purpose of palliative treatments is to increase patient's life quality, this situation affect their psychological condition.

Access to care: According to experts studying access to care, many Americans approaching the end of their lives are not able to receive hospice care. Fair and equitable access to hospice services does not exist in Egypt because the place where you live may affect the access to hospice care, for example, rural residents have less access to hospice care than the urban residents. In addition, public awareness and the training of healthcare professionals also play a role in the availability of hospice care. From my experience, I can say that very few nurses and physicians in Egypt were trained and certified in hospice and palliative care and that both rural and urban healthcare professional were not sufficiently knowledgeable about pain management, disease management and EOL issues. In addition, the difficulty of accurate prognosis may limit hospice effectiveness. Moreover, hospice programs may put additional limits on the patients if they accept, such as requiring patients to forgo specific treatments or sign a do-not resuscitate order before receiving services

Assessment of cancer patients: Many cancer specialists emphasized that they assessed their patients on the basis of performance status and their co-morbid conditions. They believed their experiences affect the patients' assessment. Some newly graduated physicians treat the disease rather than the patient especially if they work in rural areas, while experienced physicians apply special treatments for each patient, and apply their experiences in cancer patients' care, rather than relying only on text book. So, physicians who have more experience can make better decisions.

Prognosis: The ethical questions concerning prognosis include: accuracy, developing an accurate prognosis is difficult to do considering the unpredictability of disease, the large number of life-extending technologies available, and the great number of unknown and unmeasureable variables that influence how and when a person will die. Prognosis communications with patient, asking a physician to make a prognosis and inform the patient of this prognosis mostly unethical, and the patient does not embrace full and open discussion between doctors and patients about either health status or death.
End stage definition: Some of the patient's relatives ask about how long the patient will survive. So, physicians avoid answering this question because it is hard to accurately define end stage, as they would not be able to determine the patients' precise time of death, since many factors affect the patients' survival, thus each patient should be assessed separately according to their condition.

The aim of cancer therapy: Treatments for cancer patients can be classified into two main categories; palliative and curative. Most of the time, physicians do not treat cancer to increase the patient's survival, but they only treat the patient to improve their QOLe. So, the purpose of palliative treatments is to increase patient's life quality. At a stage which they know that the disease is not curable, they resort to palliative treatments.

Pain management: As a patient with serious illness approaches the EOL, symptoms, including pain, may intensify. A major part of symptom relief is the use of drugs to relieve pain, soothe anxiety, and encourage rest. Due to continuous pain patients may undergo unhappy death. Family members are repentant with sorrow due to miserable death of their loved one. Pain relief can be successfully achieved by the scientific and holistic approach of analgesic administration in palliative care. Unfortunately, some of patients still die in pain and few of these patients experience severe pain during the last days of their lives. Lack of knowledge and skills in pain assessment, improper medication, and unavailability of morphine and fear of opioid addiction are some of the challenges of palliative care. There is fear that drugs (sedatives and opioids) prescribed in the terminal stage hasten the death process. Fears of addiction to narcotics plays a key role in how people view pain management. Physicians are sometimes worried of legal and criminal scrutiny and punishment from prescribing narcotics excessively or to the wrong person. Fear of overdosing and hastening death in terminally ill patients may be unfounded However, fear of overdose and criminal punishment still remain and may lead many physicians to under-prescribe drugs.

Withholding and withdrawing medical treatment: When seriously injured or ill and approaching death, medical interventions may save or improve the life of a patient. Most people die in hospitals who are in long-term care facilities, and a majority of deaths in these settings involve withholding or withdrawing at least one of the medical treatments. Therefore, this issue will likely affect many people as they make decisions for themselves, a family member, or a loved one. Nearly many numbers of seriously ill patients are unable to speak for themselves when the time comes to decide whether or not to limit treatment.

Resuscitation: Resuscitation treatments and technologies restore and maintain breathing and heart function. However, cardiopulmonary resuscitation (CPR) doubles a person's chance of survival from sudden cardiac arrest, which is the leading cause of death in adults. While CPR is valuable for treating heart attacks and trauma, using CPR towards some dying patients may 
be inappropriate and cause complications. For some terminally ill patients, CPR is an unwanted procedure. However, the universal use of CPR makes it difficult for health professionals to stop using CPR with dying patients. Patients who do not wish to receive CPR may seek a do-not-resuscitate (DNR) order from their doctor. Family members of patients who cannot speak for themselves may also seek a DNR order on their relative's behalf. It may be easier for patients and families if physicians initiate the discussion about a DNR order. Physicians should talk with patients who are at risk of cardiopulmonary arrest (or that patient's healthcare decision-maker) and learn about their wishes regarding resuscitation, which the physician then has an ethical obligation to honor. Whether or not it is ethical to apply CPR to all patients who will stop breathing, some physicians do and others don't, it depends on personal valued. One argument suggests that DNR orders would not be necessary if CPR was limited to those cases where it is a potentially beneficial treatment.

Mechanical ventilation: It uses a machine to inflate and empty a patient's lungs allowing oxygenation of the blood. Mechanical ventilation is delivered through tubes inserted through the nose or mouth into the trachea, or through non-invasive ventilation (NIV) where air is delivered with a mask. The majority of dying patients experience breathlessness, or dyspnea, and they die. The feeling can be uncomfortable to patients and frightening for loved ones to witness. Ventilation may be given to these patients, not to extend life but to help with breathlessness. Ventilation may help them to sleep better, experience less anxiety, and eat and drink more comfortably. Mechanical ventilation is the most common life support treatment withdrawn in anticipation of death. Mechanical ventilation is such a common treatment at the EOL, that some care providers may regard mechanical ventilation as "death-delaying" rather than "life-prolonging." Some patients become dependent on the ventilator or die.

Nutrition and Hydration: Patients who are unconscious or cannot swallow can be treated through providing nutrients and water.

Enteral nutrition with feeding tubes: Delivers nutrients directly into a patient's stomach or intestines with a feeding tube. Feeding tubes are either gastrostomy tubes inserted into the stomach through an incision in the abdomen or nasogastric (NG) tubes inserted through the nose and esophagus into the stomach.

Parenteral nutrition: Delivers nutrients directly into the bloodstream. One such treatment, total parenteral nutrition (TPN), can supply a patient with nutrients to maintain his or her body weight over a long period of time.

So, nutrition and hydration can not be withholding, because food and water is similar to the act of killing a patient or allowing a person to die. A person cannot live without food and water. If nutrition and hydration are withheld or withdrawn the patient will die within a few days, even in practice, if health professionals and loved ones considered providing nutrition and hydration is to extend life will be beneficial or burdensome to the patient. A dying patient receiving nutrition and hydration via artificial way may not be ethically withheld or withdrawn whether or not the patient will die as a result of this action.

Antibiotic treatments: Dying patients are susceptible to infection. Many patients with life-threatening diseases, infection will affect their final days, and antibiotics may be given as a result. Antibiotic treatments may not cure an underlying cause of illness, but rather alleviate symptoms. ${ }^{20}$ Physicians often find it difficult to withhold antibiotic treatment from patients. One ethical concern raised by health professionals is that excessive use of antibiotics may cause mutation of bacteria, which become resistant to treatments, they express concern that over-prescribing antibiotics may result in resistant bacteria that could be more harmful to the future of the patients, particularly in light of evidence that antibiotics may not be effective for treating infection in terminally ill patients.

Euthanasia: Euthanasia is an act where a third party, usually implied to be a physician, terminates the life of a person-either passively or actively. People who are kept alive without hope of recovery for lengthy periods of time can cause their loved ones much grief, survive in a state that the patient would not have chosen or wanted, and their care can cost a great deal of money. ${ }^{21}$

Passive euthanasia allows a patient to die by stopping or refraining from beginning some type of medical intervention. For example, withholding ventilator support for breathing may be considered an act of passive euthanasia because the person would die on his or her own without the ventilator. Discontinuing dialysis is another example. Passive euthanasia is often thought of as a "allowing a person to die" because while the action of the physician removes the supportive treatment, the life-threatening illness or medical situation actually ends the patient's life.

Active euthanasia refers performing some action that terminates the life of a person. An example of an active euthanasia intervention would be a situation if a physician would inject lethal dose of a drug to a patient. ${ }^{22}$

Some physicians withdraw the treatment procedure from cancer patients at end of their life, they considered withdrawing a treatment as one of the most difficult decisions in treating cancer patients. While others don't be able to withdraw the patient's treatment themselves just because they are end stage patients. Another insisted to maintain patient's hope and they don't let the patient think they are leaving them alone to die.

There is an impression that palliative care is confused with euthanasia, which is totally forbidden by Islam. Palliative care is the moral and ethical alternative to euthanasia. Providing comfort, relieving distress, controlling pain, and offering a service that is available 24 hours a day, sustains the patient's hope. It is not based on the false hope of providing inappropriate and ineffective treatments. This society quite rightly sets great store 
on hope, and palliative care increases that hope, that each day may be more comfortable than the last. This act is Islamically forbidden because it encompasses a positive role on the part of the physician to end the life of the patient and hasten his death via lethal injection, electric shock, a sharp weapon or any other way. This is an act of killing, and, killing is a major sin and thus forbidden in Islam, the religion of pure mercy."

"Islamic jurisprudence, based on a convincing interpretation of the holy Koran, does not recognize a person's right to die voluntarily. The Islamic arguments against euthanasia can be summarized in two main reasons: Life is sacred and euthanasia and suicide are not included among the reasons allowed for killing in Islam". And Allah decides how long each of us will live and two verses support this reason. According to Islamic teachings, life is a divine trust and can not be terminated by any form of active or passive voluntary intervention. All the Islamic scholars regard active euthanasia as forbidden (Hiram) and there is no difference between Sunni and Shiite schools.

The moment of death, ajal, is under the control of Allah and the human has no say in this matter; the human can not and should not attempt to hasten or delay the ajal. The prohibition on life applies equally well whether for self, suicide, or others, homicide or genocide. The concepts of autonomy, freedom and individual choice is not applicable here for these two reasons: a. life does not belong to the human; and $b$. taking life will cause harm to the family and society in general. An individual's freedom of choice is constrained by the harm it causes to others. As a conclusion we can say that the Islamic concept is that life belongs to Allah. It is He who gives and takes away life. No human can give or take it. Muslims are against euthanasia. They believe that life of human is sacred because it is given by Allah, and that Allah chooses how long each person will live. Human beings should not interfere in this. ${ }^{23}$

Clarifying influencing factors and barriers: Discussions with patients over options would be meaningless without adequate knowledge of the medical facts (e.g., discussion on benefits of whole brain radiotherapy cannot take place unless one is cognizant of the benefits and risks in a patient with brain metastases), study of the possible barriers (e.g. if intensive care support is not available, it makes no sense to offer it to a patient who is terminally ill) and understanding of individual characteristics of the patients (e.g., if the patient's religion dictates that artificial nutrition is an obligatory act, insertion of a feeding tube would probably be non-negotiable)

Financial consideration and cost of treatment: EOL care is a significant cost to payers and patients. Healthcare and oncology drugs are very expensive; the financial and economical problems and lack of optimal insurance system are one of the most important challenges that can affect patients and their family at the time of decision-making. Discussions about the cost of treatments and care of end stage cancer patients can be of paramount importance. Consequently, considering differences of healthcare services in various cultural backgrounds and social structures, the professionals in service face numerous ethical problems for decision-making.

Most physicians would choose the standard treatment on the basis of scientific evidence and the high cost of treatment would not change their decisions. So, they may have to discuss with their patients and families about financial issues whereas they cannot avoid effective interventions on the basis of cost and they are responsible to advocate for their patients. Actually, the decision-making in this situation will be very difficult and distressful for our physicians. Financial problems are also a challenge, which can affect physicians' practice in regards with decision-making as they cannot tell patients that some treatments are more beneficial but more expensive. Furthermore, healthcare and insurance system face many challenges in regards with terminal illness and healthcare providers and planners need appropriate resources and programs to improve the quality of EOL care.

Challenges may also arise due to lack of optimal insurance system. The insurance system does not currently pay all of the oncology drugs' costs because the existing insurance coverage is not optimal, patients and their families have to pay out of the pocket to undergo these high expenditures treatments or to deprive themselves of some treatment options. In fact, if our patients can use an appropriate insurance coverage and governmental financial support, they can enjoy better treatment services and our physicians endure less distress.

Psychological, social, and cultural challenges: The development of palliative care has been limited by additional human factors. Both hospice and palliative care have come to be associated with the EOL. Psychologically most people fear and avoid things related to death. There is often a belief that even acknowledging the possibility that one may die soon is harmful. There is no evidence to support this belief and recent evidence points to the possibility that palliative care may actually improve survivality. In our country, informing patients about their diagnosis and prognosis under life threatening illness is prohibited or strongly discouraged. Previously this had been the case in most cultures, but views have been changing in the past several decades to the point where there is now some acceptance of the idea that patients themselves should be given the choice as to whether to receive this information. Life-threatening illness can have a major impact financially. In our country effective medical treatment may be available to those with the financial resources to pay for care.

Other aspects of the dying process: Patients who reach the end stage of advanced illness may also suffer from a variety of complications, including anorexia, weakness, and sexual dysfunction. Multiple interacting symptoms contribute to suffer (which is manifested by depression, cognitive disturbance, and interpersonal and spiritual crises) at the EOL. Furthermore, dying patients and their families often have to deal with complicated 
"practical" issues, such as financial problems (e.g., paying for home care) and legal issues (e.g., organizing wills or healthcare proxies). These seemingly mundane concerns can cause a great deal of distress for dying patients and their families. Physicians should be aware of the possibility that these problems may contribute to patients' distress and should be mindful of asking about them. Lastly, in those who are actively dying (i.e., the rapidly evolving process immediately preceding death, which can be recognized hours to days before death), discomfort may be heralded by shortness of breath (as a result of an accumulation of respiratory secretions).

Families involved with EOL care: Increasingly, it is recognized that "cancer is not only an individual quest; it is a family and support experience. The degree and type of family involvement, however, varies across cultures. In addition, healthcare providers' expectations regarding the participation of patient and family members during decision-making in case of cancer, also differs among cultures. In our society, the family unit is the structural foundation of Egyptian society. The impression is that patients can cope better with a terminal illness in their home, than happens elsewhere in other countries. This is probably because of the close family bonds and their strong Islamic faith with its obligation to provide for parents in case of need, to help make their lives as comfortable as possible, and think such beliefs can help them during hard times. The family is much more implicated in the cancer experience, that it seems like family's disease. In many situations, many families ask physicians for the patient being unaware of their disease. Therefore, physicians encounter ethical dilemmas and cannot decide easily, as they are obliged to fulfill the request of patient' families/relatives. In this situation, physicians have to tell the truth to the firstdegree relatives of patients, and give necessary information to the patient's family. Sometimes, patients and their family tend to take their patient home to do EOL care at home, because they want their patient to die at home; they do not like death in hospital at all, this is their family belief and they won't be happy to leave their patients in hospital. In a study reported by Alsirafy et al in 2010, described the death place of patients with advanced cancer had been referred to an Egyptian palliative care program over 1 year. Out of 79 patients, $73 \%$ died at home and $27 \%$ in hospital or ambulance. It is the authors' impression that preferences of Egyptian patients with cancer and their caregivers and the degree of social support had contributed significantly to this relatively. ${ }^{24}$

\section{Challenges Faced by Patients and Families/Healthcare Profes-} sionals Involved with EOL Care

Preferences and practices of truth telling: Truth-telling is a central concern for staff when carers act intentionally or unintentionally to prevent staff being truthful with patients. The cultural background of our society and the families' requests are the main obstacle for truth-telling. Some physicians cannot directly able to tell many patients about their serious condition, and cannot be able to inform them clearly and frankly in this regard.
This step is challenging as well as important for the physician because informing the patient about his problem helps him to be prepared to face it.

Patient's capacity is the main element of autonomybased decision-making which may be influenced by different internal factors, e.g., mental capacity, stress, and level of understanding of medical information and external factors e.g., culture, and socioeconomic class. Although family request of "do not tell" the truth and their strong resistance towards informing patients about their diseases may confuse healthcare professionals, it may also reflect divergence in patients' interests in people.

Physicians sometimes are scared to communicate and to share bad news to the patient because they may make the patient feel hopeless and unable to cope with the problem. Words like 'cancer' may create a sense of death and despair in patients. Sometimes a diagnosis of a malignant disease results in the deterioration of patient's QOL. It depends on the attitude and practice of healthcare teams about whether and how to disclose diagnoses to patients with terminal illness. Some patients will know their diagnosis without direct disclosure by the physician or their family, so not telling the truth may ruin the trust between patient and physician. Thought it is not necessary to reveal the whole truth. Thus, the patients should become aware of the required information based on their wishes. So, information about an end stage diagnosis should only be told by patients' relatives in a longer time. Some patients prefer to receive information gradually depending on their emotional responses.

Some of our physicians think that providing patients with unnecessary information would result in loss of patients' hope but also deviate the treatment plan. Most of our physicians are reluctant to tell the truth as they were not ready for such interaction. These providers consider sensitive communication a priority and make time to integrate communication into patient care. Usually, our physicians are likely to make full disclosure about a terminal illness to the patient's family, who will then decide what to tell the patient about his or her condition. Our physicians emphasized that they have not been received any formal training in key communication skills, truth-telling and breaking bad news that are very essential for EOL. Physicians should know that total information disclosure is not helpful for all patients at particular times during their illness and they should take patients' needs into account.

Physician value: It is important for a doctor to be aware of his own values and the values that drive others and their behavior. Values are pivotal to the art of medicine, and practice based on unexamined values often leads to confusion, indecision and inconsistency. Decisions regarding terminal patient care may be altered by a physician point of view and subjective attitudes toward dying patients. Physician's anxiety towards death and their discomfort with dying patients reflect on their own past experiences, both personal and professional, impact on their 
feelings regarding the treatment options available to a given patient. Some Physicians picture the situation worse than it is in order to get the patient to decide what the physician feels to be in his/her best interests. Goal is to hope for the best course of illness or for best QOL for the longest possible time

Multidisciplinary team working: Oncology is a very complicated field, which needs coordination and cooperation among multidisciplinary team working. Advanced cancer patients should be treated in a multidisciplinary approach, and a group of specialists should be involved in treating these patients. However, this approach is not possible in most of our hospitals because our centers are designed for treatment and are not appropriate for providing supportive care. Physicians and nurses are usually responsible for most of patients' needs in most of our hospital, while all of them are not in their job description. Most of our oncology hospitals are for treatment of patients, not for EOL care.

\section{Challenges Faced by Healthcare Professionals Involved with EOL Care}

Organizational responsibility: Healthcare organizations are responsible for using strategies to promote an organizational ethical climate. There is evidence that ethics protocol, guidelines and programmes may help reduce ethical conflicts. ${ }^{25,26}$ In this regard, having 'Do Not Resuscitate', 'Advance Care Planning' and communication policies and processes are important in EOL care. Evidence-based guidelines on transfer of patient to the intensive care unit (ICU) and use of welfare funds also take the burden of decision-making off staff and decrease the stress associated. Where the complexities of the case exceed those of the managing teams, there should be access to clinical ethics consultation and staff support schemes. Considering the investment in time often required to handle these situations compassionately, there is also a responsibility to ensure that the organization is adequately resourced.

Inadequate training: The oncologists believe that another reason, which creates contradictions for them and make them face difficult situations of decision-making, is educational constraints regarding medical care and ethical decision-making in courses of medicine, and even in residency courses that they have passed. Physicians who did not receive education in this field creates conflict itself. Education can be very effective; even educating specialists is very important.

The management of EOL care and the process of engaging in difficult conversations are topics that are frequently neglected in medical education. Many medical and surgical residents (who are often the physicians responsible for eliciting a patient's treatment preferences at the EOL and for facilitating difficult conversations with families) receives inadequate preparation for these tasks. Statistical studies revealed that there are particularly distressing when compared with similar studies conducted on medical instruction of invasive procedures (e.g., arterial puncture, central venous line placement, and thoracocentesis). Conventional teaching methods for invasive procedural skills involve planning ahead, demonstrating the procedure, observing the learner in action, providing feedback, and encouraging the learner's self-assessment. However, certain medical specialties (e.g., oncology and intensive care) seem to offer stronger preparatory programs for EOL care and encourage the recognition of death as part of the life cycle.

Physicians often lack the skills to communicate with patients and their families. There is a need for good planning in regards with terminal cancer which requires improvement of physicians' communication skills, provision of appropriate services for EOL care, developing multidisciplinary team working and optimal health system, and to develop guidelines for ethical decision-making and set educational priorities for physician working in this field.

Insufficient compensation: An issue that receives little formal attention among clinicians who care for dying patients is the way they are compensated for their work. Physicians who care for terminally ill patients and their families are often driven by an altruistic need to ease suffering. However, for many, altruism eventually confronts financial realities. It is reasonable to assume that continuing disparities in compensation between physicians who spend much of their time talking to patients and families and those who spend much of their time performing procedures may lead physicians who currently care for dying patients to grow resentful and keep talented individuals from entering the field. It is well known that the Medicare resource-based relative value scale compensates physicians more for invasive procedures than for evaluation and management procedures. Evaluation and management procedures include the extensive discussions with patients and families that are frequently demanded of clinicians who provide EOL care.

Personal discomfort with death: Many physicians, after intensive training that focused heavily on science and technology, feel uncomfortable when directly addressing issues (particularly those concerning spiritual and emotional needs) faced by patients and families at the EOL. Attention to dying patients tends to focus on tangible questions directed toward a nurse such as "Are they eating?" or "How are they sleeping?" rather than on questions directed toward the patient like "How are you doing?" and "What would you like to do? Clinicians frequently feel uncomfortable confronting death because they relate to and identify with terminally ill patients and their families and resist doing so in the name of professionalism. Additionally, a clinician's personal anxiety about death and disease may be further incited by interactions with a dying patient. In particular, doctors often think of death as a sign of failure or as an enemy rather than as a natural and universal part of the life process. Furthermore, a patient's dying process may remind the clinician of similar losses suffered in his or her own personal life, leading the physician to 
have surprisingly strong feelings toward this particular patient.

Ethical issues after death: After someone dies, loved ones may have to consider one or more of the following issues: Organ donation: when a person dies, the medical provider may consult the person's healthcare directive or their driver's license for their organ donation wishes. In the absence of these documents, the healthcare professional may ask a family member for permission to use the organs for organ donation. Solid organs and tissues may then be removed from the body and given to an ailing person. Family members may also be asked if the body of the newly deceased person may be donated to science as well.

Research with dying patients: The ethical issues around medical research conducted with terminal and palliative care patients are complex. Research review committees' opinion do not allow some researchers to access dying patients because the patient is already overburdened with a terminal disease and the approach of death. Others consider research with dying patients a necessary evil, and may consider it unfair to specially classify and distinguish dying patients from other patients. This includes demonstrating informed consent where a patient feels that participation is voluntary and fully understands what he or she is being asked to do who can present challenges to researchers when suggesting research to a review committee. These patients may be seen as particularly vulnerable because often they are reliant on the healthcare system and experience great amounts of emotional and physical stress.

\section{CONCLUSIONS}

The care of dying patients is fraught with challenges for patients, their loved ones, and their physicians. Clinicians must keep in mind the difficulties a patient faces in this process as well as their own professional and personal obstacles to performing this work to the best of their ability. Therefore, EOL care is optimized when approached as a thoughtful collaboration between patients, their loved ones, and their treatment teams. Clinicians are given a remarkable opportunity to help ease the physical and emotional suffering of patients and families confronting terminal illness and death. Culture and religion at least partially affect one's perception of palliative care and the decision-making that occurs at EOL. It is important to realize that there is an entire system at play that includes the patient, their family, their physicians, and other healthcare providers. In order to provide the best possible care to patients and families in EOL situations, it is important to understand their cultural constructs as well as their individual preferences. It is also important for each caregiver to realize that we each bring our own set of biases to these discussions based on our cultural and religious background and personal experiences.

\section{RECOMMENDATIONS FOR HEALTHCARE PROFESSIONALS}

1. Healthcare professionals need to be empowered with the capacity, skills, and knowledge to respond to the unique needs of each patient and their loved ones.
2. Proper cultural assessment of each patient for appropriate approach to assist them in their decision-making process.

3. Teaching and training in cultural competence among oncologists are lacking, and should be mandatory.

4. There is a need to develop culturally competent healthcare systems to address effectively different social, racial, and cultural realities of which they provide care.

5. Physicians must be aware of their own values, beliefs and attitudes towards life sustaining treatments as well as death and dying. Ethics education should be intensify especially for physicians routinely involved in EOL care.

6. Education about EOL care is very important and can improve physicians' practices in this area in order to apply these skills to patients care.

7. Physicians' response to patients who are non-compliant to appointments, prescriptions, and recommended medical care can be very ethically challenging.

\section{CONFLICTS OF INTEREST}

The author declared no conflicts of interest with respect to the authorship and/or publication of this article.

\section{REFERENCES}

1. Bullock K. The influence of culture on EOL decision making. J Soc Work End Life Palliat Care. 2011; 7: 83-98. doi: 10.1080/15524256.2011.548048

2. Heidenreich MT, Koo FK, White K. The experience of Chinese immigrant women in caring for a terminally ill family member in Australia. Collegian. 2013; 1-11. doi: 10.1016/j.colegn.2013.06.002

3. Georgaki S, Kalaidopoulou O, Liarmakopoulos I, Mystakidou K. Nurses' attitude towards truthful communication with patients with cancer. Cancer Nurs. 2002; 25(6): 436-441.

4. Molloy DW, Urbanyi M, Horsman JR, Guyatt GH. Two years of experience with a comprehensive health care directive in a home for the aged. Ann R Coll Physicians Surg Can. 1992; 25(7): 433-436.

5. Pinch WJ, Parsons ME. The ethics of treatment decision making the elderly patient's perspective. The vast resources-and costs-of available technology makes it crucial that we understand what the patient wants. Geriatr Nurs. 1993; 14(6): 289293. doi: 10.1016/S0197-4572(06)80052-2

6. Ngo-Metzger Q, August KJ, Srinivasan M, Liao S, Meskens FL. EOL care: Guide-lines for patient-centered communication. 
Am Fam Physician. 2008 77(2): 167-174.

7. Toh HC. Providing hope in terminal cancer: When is it appropriate and when is it not? Ann Acad Med Singapore. 2011; 40(1): 50-55.

8. Niezen MGH, Stolk EA, Steenhoek A, Uyl-De Groot GA. Inequalities in oncology care: Economic consequences of high drugs. Eur J Cancer. 2006; 42(17): 2887-2892. doi: 10.1016/j. ejca.2006.07.009

9. Zahedi F, Larijani B. Cancer ethics from the Islamic point of view. Iran J Allergy Asthma Immunol. 2007 6(suppl 5): 17-24.

10. Friedman Y. Ethical issues in the critically ill patient. Curr Opin Crit Care. 2001; 7(6): 475-479.

11. Field MJ, Cassel CK. Approaching Death: Improving Care at the EOL. Washington, DC, USA: National Academy Press; 1997.

12. Attree M. Patients' and relatives' experiences and perspectives of 'good' and 'not so good' quality care. J Adv Nurs. 2001; 33: $456-466$.

13. The National Hospice and Palliative Care Organization website. www.nhpco.org. Accessed May 20, 2004.

14. World Health Organization website. http://www.who.int/ cancer/palliative/en/. Accessed February 15, 2004.

15. Ruddick W. Do doctors undertreat pain? Bioethics.1997; 11(3-4): 246-255.

16. Chrystal-Frances E. Palliative care: A discussion of management and ethical issues. Nursing Forum, 2003; 38(2):25-29.

17. LaDuke S. Terminal dyspnea \& palliative care. Am J Nurs.
101(11): 26-31.

18. Weiss SC, Emanuel LL, Fairclough DL, Emanuel EJ. Understanding the experience of pain in terminally ill patients. Lancet. 2001; 357(9265): 1311-1315. doi: 10.1016/S01406736(00)04515-3

19. Barraclough J. Cancer and Emotion: A Practical Guide to Psycho-oncology. Abingdon, England: Radcliffe Medical; 1994.

20. Marcus EL, Clarfield AM, Moses AE. Ethical issues relating to the use of antimicrobial therapy in adults. Clin Infect Dis. 2001; 33(10): 1697-1705. doi: 10.1086/323757

21. The Multi-Society Task Force on PVS. Medical aspects of the persistent vegetative state- first of two parts. $N$ Engl J Med. 1994; 330: 1499-1508. doi: 10.1056/NEJM199405263302107

22. Steinbock B, Norcross A. Introduction. Killing and Letting Die. $2^{\text {nd }}$ ed. New York, USA: Fordham University Press; 1994.

23. Procon. What are muslim perspectives on euthanasia and physician-assisted suicide? Web site. http://euthanasia.procon. org/view.answers.php?questionID $=000156$. Accessed June 25, 2016.

24. Alsirafy SA, El Mesidy SM, Abou-Elela EN. Where do egyptian palliative care patients with cancer die? Am J Hospice Palliat Med. 2010; 27: 313-315. doi: 10.1177/1049909109357613

25. Nelson WA, Weeks WB, Campfield JM. The organizational costs of ethical conflicts. J Healthc Manag. 2008; 53: 41-52.

26. Bacchetta MD, Fins JJ. The economics of clinical ethics programs: A quantitative justification. Camb $Q$ Healthc Ethics. 1997; 6: 451-460. 